\title{
Heegaard splittings and the pants complex
}

\author{
JESSE JOHNSON
}

\begin{abstract}
We define integral measures of complexity for Heegaard splittings based on the graph dual to the curve complex and on the pants complex defined by Hatcher and Thurston. As the Heegaard splitting is stabilized, the sequence of complexities turns out to converge to a non-trivial limit depending only on the manifold. We then use a similar method to compare different manifolds, defining a distance which converges under stabilization to an integer related to Dehn surgeries between the two manifolds.
\end{abstract}

57N10; 57M27, 57M99

\section{Introduction}

Hempel introduced the curve complex to the study of Heegaard splittings by defining a distance which generalizes the definitions of reducible, weakly reducible and the disjoint curve property. This has proved very useful in studying irreducible splittings of manifolds. Hempel's definition of distance is, in some sense, very local. If a Heegaard splitting is stabilized only once, the distance drops to zero, regardless of the original splitting.

This definition of distance can be directly modified to use the pants complex defined by Hatcher and Thurston, or the closely related dual graph to the curve complex. (All three spaces will be defined in detail in the next section.) The two types of distance that come from these metric spaces prove to give a more global measure of complexity. In most cases, when a splitting is stabilized, the distance will increase by one (rather than dropping to zero.) Thus if the genus is subtracted from the distance, the resulting integer, called the complexity, should tend to stay constant under stabilization.

We will show that for any Heegaard splitting of a given manifold, under an infinite sequence of stabilizations the complexities of the stabilizations form a convergent sequence whose limit is non-trivial and which depends only on the manifold.

Section 2 contains the definitions of the three spaces mentioned above. In Section 3, we consider maximal collections of disks in compression bodies. This will allow us to apply the later results to manifolds with boundary as well as closed manifolds. We 
define the dual distance and pants distance in Section 4 and give lower bounds for this distance depending on the ambient 3-manifold.

In Section 5, we define the complexity of a Heegaard splitting and show that the sequence of complexities converges. The limit is called the Heegaard complexity of the manifold. The basic properties of the Heegaard complexity are examined in Section 6 .

Finally, in Section 7, we show that a similar technique can be used to define a measure of the distance between different manifolds. This distance turns out to be equal to the minimal number of components needed for a link such that Dehn surgery on the link in one manifold produces the second manifold. Section 8 is a list of questions that arise and speculations about applications.

I would like to thank my advisor, Abby Thompson, for her guidance and support. This research was supported by NSF VIGRE grant 0135345.

\section{Definitions}

Let $\Sigma$ be a compact, connected, closed, orientable surface. Throughout the paper, we will assume the genus of $\Sigma$ is greater than 1 . We will give the definitions of three metric spaces based on $\Sigma$.

The first, the curve complex, $C(\Sigma)$, is the cell complex defined as follows: The vertices of $C(\Sigma)$ are isotopy classes of non-trivial, simple closed curves in $\Sigma$. An edge will connect two vertices if and only if there are representatives of the two isotopy classes which are disjoint.

The graph can be filled in with cells of higher dimension. A collection of vertices $\left\{u_{0}, \ldots, u_{n}\right\}$ bounds an $n$-simplex in $C(\Sigma)$ if and only if some collection of loops defined by the vertices are pairwise disjoint. Let $g$ be the genus of $\Sigma$. Then $C(\Sigma)$ has dimension $3 g-4$, a maximal simplex corresponding to a pair-of-pants decomposition of $\Sigma$, with $3 g-3$ loops.

The second space, the dual curve complex, $C^{*}(\Sigma)$, will be defined as follows: each vertex $v \in C^{*}(\Sigma)$ corresponds to a maximal dimensional simplex $\sigma_{v}$ in $C(\Sigma)$, ie a pair-of-pants decomposition of $\Sigma$. We will use the convention that $u$ is a vertex of $C(\Sigma)$ and $v$ is a vertex of $C^{*}(\Sigma)$.

Two vertices, $v, v^{\prime}$ are connected by an edge if and only if the simplices $\sigma_{v}$ and $\sigma_{v^{\prime}}$ in $C(\Sigma)$ share a co-dimension one face. An edge thus corresponds to a move of the following type: Start with a pants decomposition of $\Sigma$; remove one loop and replace it with a loop which is disjoint from all the other loops and which creates a new pants decomposition.

Algebraic 83 Geometric Topology, Volume 6 (2006) 
The third space, the pants complex, $C^{P}(\Sigma)$, was defined by Hatcher and Thurston [3] as a discrete quotient of the space of Morse functions on $\Sigma$. Every Morse function on $\Sigma$ suggests a decomposition of $\Sigma$ into pairs of pants, forming the vertices of $C^{P}(\Sigma)$. Near-Morse functions, in which there are two critical points at the same level, form co-dimension one sets between the connected components of Morse functions. These suggest edges between certain vertices.

Careful analysis shows that the edges correspond to moves of the following type: Given a pair-of-pants decomposition of $\Sigma$, remove a loop from the pants decomposition and replace it with a loop which is disjoint from the other loops and intersects the original loop minimally. A minimal intersection between two loops is defined as follows: Consider a pair-of-pants decomposition $\mathbf{L}=\left\{l_{0}, \ldots, l_{m}\right\}$. The complement of $\mathbf{L}$ in
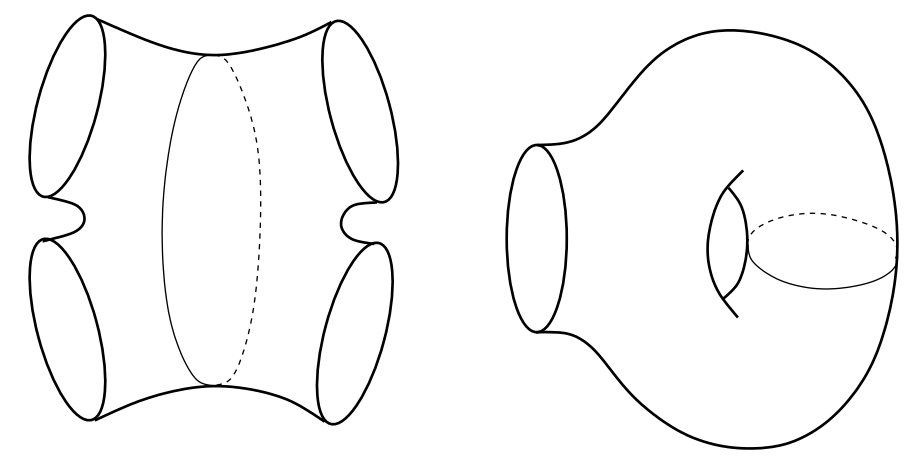

Figure 1: The two types of loops in a pair-of-pants decomposition

$\Sigma$ is a collection of pairs of pants. The complement of $\left\{l_{0}, \ldots, \hat{l}_{i}, \ldots, l_{m}\right\}$ contains a number of pairs of pants and one component, containing $l_{i}$, which is not a pair of pants. Call this component $C$. The surface $C$ is the result of either gluing two pairs together along the loop $l_{i}$ or of gluing together two cuffs of the same pair. In the first case, $C$ is a sphere with four punctures. In the second case, $C$ is a torus with a single puncture. (See Figure 1.)

A loop $l_{i}^{\prime}$, disjoint from and not parallel to $l_{0}, \ldots, \widehat{l}_{i}, \ldots, l_{m}$ must lie in $C$. If $C$ is a four-punctured sphere then $l_{i}^{\prime}$ intersects $l_{i}$ minimally when there are two points of intersection. (The loop is separating so there must be an even number of intersections.) If $C$ is a once-punctured torus, then one point of intersection is minimal.

Because the space of Morse functions on $\Sigma$ is connected, Hatcher and Thurston were able to show that the pants complex is connected. There is a canonical map from the vertices of $C^{P}(\Sigma)$ to the vertices of $C^{*}(\Sigma)$. For each edge of $C^{P}(\Sigma)$, there is a 
corresponding edge of $C^{*}(\Sigma)$ (but not vice versa) so there is a canonical embedding of $C^{P}(\Sigma)$ into $C^{*}(\Sigma)$ which is onto the vertices of $C^{*}(\Sigma)$. Since $C^{P}(\Sigma)$ is connected, it follows that $C^{*}(\Sigma)$ is connected.

Brock [1] has shown that the pants complex, $C^{P}(\Sigma)$, is quasi-isometric to Teichmuller space with the Weil-Peterson metric and that distances in the pants complex are related to the volumes of convex cores of hyperbolic manifolds of the form $\Sigma \times \mathbb{R}$. We will study the pants complex and its relative, the dual curve complex, in relation to Heegaard splittings, in analogue with Hempel's work with the curve complex [4].

Let $H$ be a handlebody and let $\phi: \Sigma \rightarrow \partial H$ be a homeomorphism. For a vertex $u \in C(\Sigma)$, write $u \in H$ if for some loop $l$ in the isotopy class corresponding to $u$, $\phi(l)$ bounds a disk in $H$. (Note: If this is true for one loop in the isotopy class, then it is true for all the loops in the isotopy class.)

An edge path between two vertices $u, u^{\prime}$ in the curve complex corresponds to a sequence of loops $u \ni l_{0}, \ldots, l_{n} \in u^{\prime}$ in $\Sigma$ such that consecutive loops are disjoint. The length of the path is $n$ and the distance $d\left(u, u^{\prime}\right)$ is the length of the shortest possible path. This is often called the geodesic metric on $C(\Sigma)$.

Let $\left(\Sigma, H_{1}, H_{2}\right)$ be a Heegaard splitting of a manifold $M$. Consider the inclusion maps $\Sigma \rightarrow H_{i}$. Each map suggests a set of vertices in $C(\Sigma)$ which are in $H_{i}$. The standard distance of $\Sigma$, as in Hempel [4], is $d(\Sigma)=\min \left\{d\left(u, u^{\prime}\right) \mid u \in H_{1}, u^{\prime} \in H_{2}\right\}$. This distance measures the irreducibility of $\Sigma$, in the sense that if $d(\Sigma)=0$ then $\Sigma$ is reducible, if $d(\Sigma)=1$ then $\Sigma$ is weakly reducible and if $d(\Sigma)=2$ then $\Sigma$ has the disjoint curve property.

There are analogous definitions for the distance of a Heegaard splitting based on the dual curve complex and the pants complex. We will define these right after a short aside about collections of disks in compression bodies.

\section{Maximal collections of disks}

A 1-handle is ball parameterized as $D \times[0,1]$, where $D$ is a disk. Let $F$ be a surface (not necessarily connected) with no sphere components. A compression body is either a handlebody or a connected manifold constructed by gluing 1-handles to the boundary component $F \times\{1\}$ of $F \times[0,1]$ along the disks $D \times\{0\}$ and $D \times\{1\}$. For a compression body $H$, let $\partial_{-} H=F \times\{0\}$ and let $\partial_{+} H=\partial H \backslash \partial_{-} H$. If $H$ is a handlebody then $\partial_{+} H=\partial H$ and $\partial_{-} H=\varnothing$.

Let $H$ be a compression body and let $\mathbf{D}=\left\{D_{0}, \ldots, D_{n}\right\}$ be a collection of pairwise disjoint, pairwise non-parallel, properly embedded disks in $H$. 
Definition 1 The collection $\mathbf{D}$ is maximal if any properly embedded disk in $H$ that is disjoint from $\mathbf{D}$ is parallel to one of the disks in $\mathbf{D}$.

If $H$ is a handlebody, a maximal collection of disks gives a pair-of-pants decomposition for $\partial H$. Otherwise, when $\partial_{-} H \neq \varnothing$, there is no collection of disks which cuts $\partial H$ into pairs of pants, so we have to be more careful. A maximal collection of disks will cut $H$ into a collection of balls and pieces homeomorphic to $\partial_{-} H \times I$. However, not every collection of disks with this property will be maximal.

Lemma 2 Let $\mathbf{D}$ be a collection of disjoint, properly embedded disks in $H$ (not necessarily maximal). The closure of the complement of $\mathbf{D}$ is a collection of balls and compression bodies.

The proof of this Lemma is left to the reader. Let $H^{\prime}$ be a component of $H \backslash \mathbf{D}$ and let $\bar{H}^{\prime}$ be its closure. Then $\bar{H}^{\prime} \backslash H^{\prime}$ is a collection of disks along which $H$ was cut. We will call a component of $\bar{H}^{\prime} \backslash H^{\prime}$ a scar on $H^{\prime}$.

Lemma 3 Let $H$ be a compression body and not a ball, a solid torus or a (surface) $\times I$ and let $\mathbf{D}$ be a maximal collection of disjoint, non-parallel essential disks. Then each component of $H \backslash \mathbf{D}$ is either a ball with three scars or a piece of the form (surface) $\times I$ with exactly one scar.

Proof Let $H^{\prime}$ be a component of $H \backslash \mathbf{D}$. From Lemma 2, we know that $H^{\prime}$ is either a ball or a compression body.

First assume $H^{\prime}$ is a ball. If there is one scar on $H^{\prime}$ then this scar corresponds to a boundary parallel disk in $\mathbf{D}$, but we assumed all the disks in $\mathbf{D}$ were essential, so this is impossible. If there are two scars on $H^{\prime}$ then there are two disks in $\mathbf{D}$ which are parallel in $H$, and again we assumed this is not the case.

If there are more than three scars on $H^{\prime}$ then $\partial \bar{H}^{\prime} \cap H^{\prime}$ is a sphere with at least four punctures. Let $l$ be an essential loop in this surface which is not boundary-parallel. Then $l$ bounds a disk in $H^{\prime}$, and therefore in $H$. This disk is properly embedded, disjoint from the rest of the disks, and not parallel to any of them. Thus the maximality assumption implies that every ball component $H^{\prime}$ must have exactly three scars.

Now assume $\bar{H}^{\prime}$ is a compression body. By definition, $H^{\prime}$ is the result of attaching 1 -handles to components of the form (surface) $\times I$. A 1 -handle would define an essential disk which is not parallel to any of the disks in $\mathbf{D}$, so maximality implies there cannot be any 1 -handles and $\bar{H}^{\prime}$ must be of the form $F \times I$ where $F$ is a surface and not a sphere.

Algebraic 83 Geometric Topology, Volume 6 (2006) 
Because $H$ is connected and $H^{\prime}$ is not all of $H$, there must be at least one scar on $H^{\prime}$. If there are two or more scars on $H^{\prime}$, let $l_{1}, l_{2}$ be the boundaries of two scars in $\partial \bar{H}$. Let $\alpha$ be an arc from $l_{1}$ to $l_{2}$, disjoint from the rest of the scars, and let $N$ be a regular neighborhood of $l_{1} \cup l_{2} \cup \alpha$ in $\partial \bar{H}^{\prime}$. The disk $\partial N \backslash \partial \bar{H}$ will be disjoint from the scars in $\partial \bar{H}^{\prime}$ and boundary-parallel in $H^{\prime}$. Because of the scars bounded by $l_{1}$ and $l_{2}$, this disk is non-trivial in $H$ and not parallel to any of the disks in $\mathbf{D}$. Thus maximality of $\mathbf{D}$ implies that $H^{\prime}$ must have exactly one scar.

Lemma 4 Let $\Sigma$ be a positive-genus surface and let $\mathbf{L}$ be a pair-of-pants decomposition for $\Sigma$. Then some loop $l \in \mathbf{L}$ is non-separating.

Proof Cutting a positive-genus surface along a separating loop produces two surfaces (with boundary), each with strictly positive genus. By induction, if we cut $\Sigma$ along all the separating loops in $\mathbf{L}$, the result will be a number of positive-genus surfaces. Since $\mathbf{L}$ is a pair-of-pants decomposition, cutting along all the loops should produce a collection of pairs of pants (genus-zero surfaces) so at least one of the loops in $\mathbf{L}$ must be non-separating.

Lemma 5 Let $\Sigma$ be a closed surface of genus $g$. Let $\mathbf{L}$ be a pair-of-pants decomposition of $\Sigma$ and let $l_{1}, \ldots, l_{n} \in \mathbf{L}$ be distinct loops such that their union does not separate $\Sigma$. Then there are loops $l_{n+1}, \ldots, l_{g} \in \mathbf{L}$ such that $l_{1}, \ldots, l_{g}$ are distinct loops whose union does not separate $\Sigma$.

Proof Given loops $l_{1}, \ldots, l_{n}$, we will show that if $n<g$ then there is a distinct loop $l_{n+1} \in \mathbf{L}$ such that the union of $l_{1}, \ldots, l_{n+1}$ does not separate $\Sigma$. By taking the collection to be maximal, this implies the desired result.

Let $\Sigma^{\prime}$ be the result of cutting $\Sigma$ along the loops $l_{1}, \ldots, l_{n}$ and gluing a disk into each boundary component of the resulting surface. Because the union of the loops $l_{1}, \ldots, l_{n}$ does not separate $\Sigma$, the surface $\Sigma^{\prime}$ is connected. We also assumed $n<g$, so $\Sigma^{\prime}$ has strictly positive genus.

The remaining loops in $\mathbf{L}$ contain a pair-of-pants decomposition for $\Sigma^{\prime}$. (Some of the loops remaining in $\mathbf{L}$ will be trivial or parallel in $\Sigma^{\prime}$ and need to be thrown out.) By Lemma 4 , there is a loop $l_{n+1} \in \mathbf{L}$ in the induced pair-of-pants decomposition which does not separate $\Sigma^{\prime}$. Then $l_{1}, \ldots, l_{n+1}$ does not separate $\Sigma$. By induction, the proof is complete.

Given a compression body $H$, let $g$ be the genus of $\partial_{+} H$ and let $b$ be the sum of the genera of the components of $\partial_{-} H$. 
Lemma 6 Let $\mathbf{D}$ be a maximal collection of disks for $H$ and let $\mathbf{L}$ be a pair-of-pants decomposition for $\partial_{+} H$ such that for each disk $D \in \mathbf{D}, \partial D \in \mathbf{L}$. If $l_{1}, \ldots, l_{g} \in \mathbf{L}$ is a collection of distinct loops whose union does not separate $\Sigma$, then at least $g-b$ of these loops bound disks in $\mathbf{D}$.

Proof Cut $H$ along the disks in D. Let $H^{\prime}$ be a resulting component of the form $F \times I$ where $F$ is a surface of genus $g^{\prime}$. Any collection of more than $g^{\prime}$ loops in $F$ will separate $F$, so if more than $g^{\prime}$ of the loops $l_{1}, \ldots, l_{g}$ are in $H^{\prime}$, then these loops will separate $\partial_{+} H^{\prime}$. Because there is exactly one scar on $H^{\prime}$, these disks would also separate $H$. Since we assumed that the union of $l_{1}, \ldots, l_{g}$ does not separate $H$, at most $g^{\prime}$ of these loops can be in $H^{\prime}$.

The same is true for any non-ball component of $H \backslash \mathbf{D}$, so the number of loops that are not boundaries of disks is at most $b$.

\section{Distance and Heegaard splittings}

We will now define a notion of distance for Heegaard splittings based on the dual curve complex. Let $v$ be a vertex of $C^{*}(\Sigma)$. We will say $v$ defines a compression body $H$ if there is a maximal collection of disks for $H$ such that the boundary of each disk defines a vertex $u$ of $\sigma_{v}$.

Lemma 7 Assume $v$ defines a compression body $H$, with $\phi: \Sigma \rightarrow \partial_{+} H$ and a second compression body $H^{\prime}$ with $\phi^{\prime}: \Sigma \rightarrow \partial_{+} H^{\prime}$. If the same vertices of $v$ bound disks in $H$ as in $H^{\prime}$ then there is a homeomorphism $\psi: H \rightarrow H^{\prime}$ such that $\psi \circ \phi=\phi^{\prime}$.

A proof of the Lemma is left to the reader. The converse is not true. In general, given two pants decompositions of $\Sigma$, there may not be an automorphism of $\Sigma$ taking one to the other. For example, if one of the pants decompositions consists entirely of nonseparating loops (such a decomposition exists) then there will be no homeomorphism taking it to a pants decomposition containing separating loops.

The dual distance $D\left(v, v^{\prime}\right)$ between two vertices in $C^{*}(\Sigma)$ is the length of the shortest path in $C^{*}(\Sigma)$ between them. For a Heegaard splitting, $\left(\Sigma, H_{1}, H_{2}\right)$, the dual distance of $\Sigma$ is $D(\Sigma)=\min \left\{D\left(v, v^{\prime}\right) \mid v\right.$ defines $H_{1}, v^{\prime}$ defines $\left.H_{2}\right\}$. Note that $D(\Sigma) \geq d(\Sigma)$. Hempel has shown that there are genus two Heegaard splittings such that $d(\Sigma)$ is arbitrarily large. Thus there are Heegaard splittings with $D(\Sigma)$ arbitrarily large.

Let $D^{P}\left(v, v^{\prime}\right)$ be the distance between vertices $v$ and $v^{\prime}$ in the pants complex. Because of the one-to-one map between the vertices of $C^{*}(\Sigma)$ and the vertices of $C^{P}(\Sigma)$, we 
can think of $v$ and $v^{\prime}$ as being in either graph. An edge path in $C^{P}$ maps to an edge path of the same length in $C^{*}$ so $D\left(v, v^{\prime}\right) \leq D^{P}\left(v, v^{\prime}\right)$.

Let $\left(\Sigma, H_{1}, H_{2}\right)$ be a genus $g$ Heegaard splitting of a 3-manifold $M$. From now on we will assume that $H_{2}$ is a handlebody (ie $\partial_{-} H_{2}=\varnothing$ ) but we will allow $H_{1}$ to be a compression body. Thus $\partial_{-} H_{1}=\partial M$ and $H_{1}$ will be a handlebody if and only if $M$ is closed. Such a Heegaard splitting always exists.

Let $b$ be the sum of the genera of the boundary components of $M$ and let $n$ be the maximal number of disjoint, embedded 2-spheres $S_{1}, \ldots, S_{n}$ such that $M \backslash\left(\bigcup S_{i}\right)$ is connected. (Equivalently, $n$ is the number of $S^{1} \times S^{2}$ components of the prime decomposition of $M$, so $n$ is well defined and finite.)

Lemma $8 \quad D(\Sigma) \geq g-b-n$

Proof Let $v$ define $H_{1}$ and $v^{\prime}$ define $H_{2}$ and assume for contradiction $D\left(v, v^{\prime}\right)=$ $D(\Sigma)<g-b-n$. Let $\mathbf{L}$ be a collection of pairwise-disjoint loops in $\Sigma_{g}$ corresponding to the vertices of $\sigma_{v}$. Recall that $H_{1}$ may be a compression body (if $b>0$ ) or a handlebody (if $b=0$ ).

By Lemma 5 , there are loops $l_{1}, \ldots, l_{g} \in \mathbf{L}$ whose union does not separate $\Sigma$. Each step in the path from $v$ to $v^{\prime}$ changes one vertex. Since $D\left(v, v^{\prime}\right) \leq g-(b+n+1)$ and there are $g$ loops in the collection, at least $b+n+1$ of the loops $l_{1}, \ldots, l_{g}$ correspond to vertices of $\sigma_{v^{\prime}}$. Since $\mathrm{H}_{2}$ is a handlebody, these $b+n+1$ loops bound disks in $H_{2}$. By Lemma 6 , at most $b$ of the loops do not bound disks in $H_{1}$ so at least $n+1$ of the loops $l_{1}, \ldots, l_{2}$ bound disks in both $H_{1}$ and $H_{2}$.

Let $l_{1}, \ldots, l_{m}$ be the loops that bound disks in both $H_{1}$ and $H_{2}$. Then each $l_{i}$ defines an embedded sphere $S_{i}$. These spheres are disjoint and because the loops $l_{1}, \ldots, l_{m}$ do not separate $\Sigma$, the spheres $S_{1}, \ldots, S_{m}$ do not separate $M$ (hence they are nonparallel). We assumed that $M$ contains at most $n$ such spheres, so we must have $m \leq n$. However, we showed that there are at least $n+1$ loops bounding disks on both sides. This contradiction completes the proof.

Corollary 9 If $M$ is closed and irreducible, then $D(\Sigma) \geq g$.

Lemma $10 D^{P}(\Sigma) \geq g-b-n$

The proof of Lemma 10 is identical to the proof of Lemma 8, after replacing each $D$ with $D^{P}$.

Lemma 11 If $D(\Sigma)=g-b-n$ then $M=S^{3}$ or $M$ is a connect sum of lens spaces, handlebodies and copies of $S^{1} \times S^{2}$. 
Proof Let $v, v^{\prime}$ be vertices of $C^{*}(\Sigma)$ that define $H_{1}$ and $H_{2}$, respectively, so that $D\left(v, v^{\prime}\right)=D(\Sigma)=g-b-n$. As in the previous proof, let $\mathbf{L}$ be the collection of loops corresponding to the vertices of $\sigma_{v}$ and let $l_{1}, \ldots, l_{g} \in \mathbf{L}$ be a collection of loops whose union is non-separating.

We saw that if more than $b+n$ of these loops also correspond to vertices of $\sigma_{v^{\prime}}$ then we have a contradiction. Because $D\left(v, v^{\prime}\right)=g-b-n$, and there are $g$ loops, at least $b+n$ of them must be common to $v$ and $v^{\prime}$ so we know that exactly $b+n$ of the loops $l_{1}, \ldots, l_{g}$ are common to both $\sigma_{v}$ and $\sigma_{v^{\prime}}$ and each of the remaining loops is moved exactly once. The remaining loops of $\mathbf{L}$ are not moved.

Of the $b+n$ loops that are common to $\sigma_{v}$ and $\sigma_{v^{\prime}}$, at most $n$ bound disks in $H_{1}$ (because there are at most $n$ non-separating spheres in $M$ ) so at least $b$ of the loops do not bound disks in $H_{1}$. Thus each of the loops in $l_{1}, \ldots, l_{g}$ that is moved must bound a disk in $H_{1}$.

Assume $l_{1}$ is the first loop that is moved, and replaced by a loop $l_{1}^{\prime}$. Since $H_{2}$ is a handlebody and the loop $l_{1}^{\prime}$ is not moved later in the path in $C^{*}(\Sigma), l_{1}^{\prime}$ must bound a disk in $H_{2}$. Recall that there are two types of loops in the pants decomposition, defined by whether removing the loop from the collection produces a four-punctured sphere or a once-punctured torus.

If $l_{1}$ is of the first type, then the four-punctured sphere is part of the boundary of a ball in $H_{1}$. Since $l_{1}^{\prime}$ sits in the four-punctured sphere, it bounds a disk in the ball in $H_{1}$, and therefore bounds a disk in $H_{1}$. Thus we could have started the path with $l_{1}^{\prime}$ in the pants decomposition instead of $l_{1}$. Because the path in $C^{*}(\Sigma)$ was assumed to be minimal, $l_{1}$ must sit in a punctured torus.

Let $l_{1}^{\prime \prime}$ be the loop defining the boundary of the puncture. Notice that $l_{1}^{\prime \prime}$ is separating, so it cannot be one of the non-separating loops $l_{1}, \ldots, l_{g}$ and it cannot be moved later on. In particular, this implies that $l_{1}$ cannot be adjacent to any of the loops that are moved later in the path.

The loop $l_{1}^{\prime \prime}$ bounds a disk in $H_{1}$ and a disk in $H_{2}$, so $l_{1}^{\prime \prime}$ defines a sphere in $M$ which cuts off a genus-one piece of the Heegaard splitting. Thus the sphere defines either a stabilization or a connect sum with a lens space.

Assume $l_{2}$ is the next loop which is moved. We saw that $l_{2}$ cannot be adjacent to $l_{1}$, so $l_{2}$ sits in a punctured torus, with a punctured bounded by $l_{2}^{\prime \prime}$. Again, $l_{2}^{\prime \prime}$ defines a sphere which separates a genus-one piece of the Heegaard splitting, so $l_{2}^{\prime \prime}$ defines a stabilization or a connect sum with a lens space. Continuing in this fashion for each loop that is moved, we get a collection of $D(\Sigma)$ stabilizations and lens space summands. 
Let $M^{\prime}$ be the result of cutting $M$ along these spheres and gluing balls into the resulting boundary components. Let $\left(\Sigma^{\prime}, H_{1}^{\prime}, H_{2}^{\prime}\right)$ be the Heegaard splitting resulting from gluing disks into the image of $\Sigma$ in $M^{\prime}$. Since we removed from $M$ all the loops that were not in both the pants decomposition of $H_{1}$ and that of $H_{2}$, it follows that $D\left(\Sigma^{\prime}\right)=0$.

Let $F$ be a component of $\partial_{-} H_{1}$. Because the pants decomposition for $\Sigma$ was maximal, there is a loop $l^{\prime \prime}$ which bounds a disk in $H_{1}$ and separates $H_{1}$ into a compression body and an $F \times I$ component. Since $D\left(\Sigma^{\prime}\right)=0$, the loop $l^{\prime \prime}$ bounds a disk in $H_{2}$ and defines a separating sphere in $M^{\prime}$. The disks cut off a handlebody from $H_{2}$ and a $F \times I$ component from $H_{1}$, so the sphere cuts off a handlebody summand from $M^{\prime}$.

For each boundary component of $M^{\prime}$, (each corresponding to a component of $\partial_{-} H_{1}$ ) there is a corresponding loop defining a sphere which cuts off a handlebody summand from $M$. Let $M^{\prime \prime}$ be the result of cutting $M^{\prime}$ along these spheres and gluing balls into the resulting spheres. Let $\left(\Sigma^{\prime \prime}, H_{1}^{\prime \prime}, H_{2}^{\prime \prime}\right)$ be the Heegaard splitting resulting from repairing the image of $\Sigma^{\prime}$ in $M^{\prime \prime}$.

If $H_{1}^{\prime \prime}$ is a ball then $M^{\prime \prime}$ is $S^{3}$ and we are done. Otherwise, $M^{\prime \prime}$ is closed and $D\left(\Sigma^{\prime \prime}\right)=0$, so $M^{\prime \prime}$ is the result of gluing together two handlebodies by the identity map on their boundaries. This construction always yields a connect sum of copies of $S^{1} \times S^{2}$. This completes the proof.

Lemma 12 If $D^{P}(\Sigma)=g-b-n$ then $M=S^{3}$ or $M$ is a connect sum of handlebodies and copies of $S^{1} \times S^{2}$.

Again, the proof of this lemma is almost identical to the analogous proof for $D(\Sigma)$. Note, however, that the set of manifolds in the second lemma is more restricted.

\section{Stabilization}

Let $\left(\Sigma, H_{1}, H_{2}\right)$ be a Heegaard splitting of $M$ with $b$ and $n$ defined as in Section 4. For each $g$ greater then or equal to the genus of $\Sigma$, let $\Sigma_{g}$ be a stabilization of $\Sigma$ such that $\Sigma_{g}$ has genus $g$. In other words, $\Sigma_{g}$ is the result of attaching zero or more trivial handles to $\Sigma$ so that the resulting surface is a Heegaard surface.

Define $A_{g}(\Sigma)=D\left(\Sigma_{g}\right)+b-g$ and $A_{g}^{P}(\Sigma)=D^{P}\left(\Sigma_{g}\right)+b-g$. We will consider the limiting behavior of these two values. From Lemmas 8 through 12, the following two Lemmas follow immediately:

Lemma $13 A_{g}(\Sigma) \geq-n$ and if $A_{g}(\Sigma)=-n$ then $M=S^{3}$ or $M$ is a connect sum of handlebodies, copies of $S^{1} \times S^{2}$ and lens spaces. 
Lemma $14 A_{g}^{P}(\Sigma) \geq-n$ and if $A_{g}(\Sigma)=-n$ then $M=S^{3}$ or $M$ is a connect sum of handlebodies and copies of $S^{1} \times S^{2}$.

This gives us a lower bound on the sequences $A_{g}(\Sigma)$ and $A_{g}^{P}(\Sigma)$ as $g \rightarrow \infty$. We will next show that the sequences are also bounded above. For the following lemmas, consider a fixed $g$ and a sequence of stabilizations $\Sigma_{h}$.

Lemma 15 If $h>g$ then $A_{h}(\Sigma) \leq 2 A_{g}(\Sigma)+g-b$ and $A_{h}^{P}(\Sigma) \leq 2 A_{g}^{P}(\Sigma)+g-b$.

Proof We will prove that $D\left(\Sigma_{h}\right) \leq 2 D\left(\Sigma_{g}\right)+(h-g)$. By subtracting $h$ from both sides we get the stated result. The pants distance, $D^{P}$ can be substituted for $D$ throughout the proof. Let $v_{1}, \ldots, v_{n}$ be a minimal path from $H_{1}$ to $H_{2}$ in $C^{*}(\Sigma)$ (or $\left.C^{P}(\Sigma)\right)$. Let $l_{1}^{1}, \ldots, l_{m}^{1}(m=3 g-3)$ be loops in $\Sigma$ corresponding to the vertices of $\sigma_{v_{1}}$.

For each $v_{i}$, let $l_{i}^{1}, \ldots, l_{i}^{m}$ be loops corresponding to the vertices of $\sigma_{v_{i}}$ and assume the loops are labeled so that if $l_{i-1}^{j}$ is a vertex of both $\sigma_{v_{i-1}}$ and $\sigma_{v_{i}}$ then $l_{i}^{j}=l_{i-1}^{j}$. In other words, if the move from $v_{i-1}$ to $v_{i}$ replaces a loop $l_{i-1}^{j}$ with a new loop, this new loop is labeled $l_{i}^{j}$. If a loop $l_{i-1}^{j}$ is not replaced, the same loop appears in $v_{i}$ as $l_{i}^{j}$
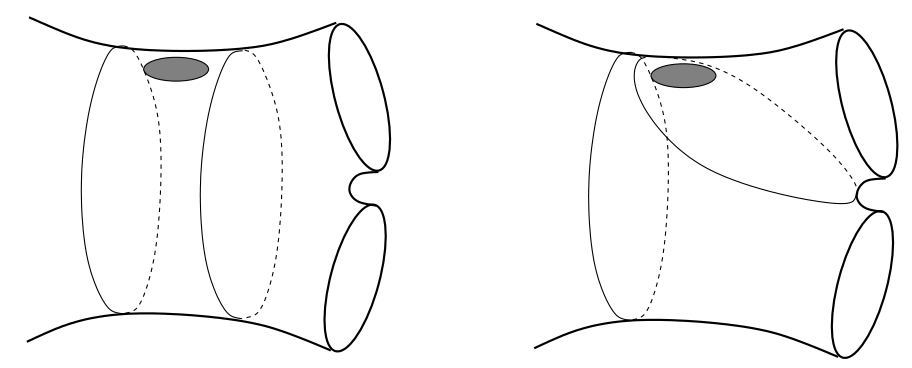

Figure 2: One extra move is required to get the extra loop out of the way.

We will define a stabilization of $\Sigma_{g}$ and a pants decomposition $k_{1}^{1}, \ldots, k_{1}^{m^{\prime}}\left(m^{\prime}=\right.$ $3 h-3$ ) as follows: Let $k_{1}^{j}=l_{1}^{j}$ for $1 \leq j \leq m$. Let $k_{1}^{m+1}$ be a loop parallel to $k_{1}^{1}$ and let $k_{1}^{m+2}$ be a trivial loop in the resulting annulus. See Figure 2.

Puncture $\Sigma$ in the disk defined by $k_{1}^{m+2}$ and construct a new surface $\Sigma^{\prime}$ by attaching a punctured genus- $(h-g)$ surface to $\Sigma$ (so that the resulting surface is a stabilization). Let $k_{1}^{m+3}, \ldots, k_{1}^{m^{\prime}}$ be loops on $\Sigma^{\prime}$ as shown in Figure 3 and let $\left(\Sigma^{\prime}, H_{1}^{\prime}, H_{2}^{\prime}\right)$ be 


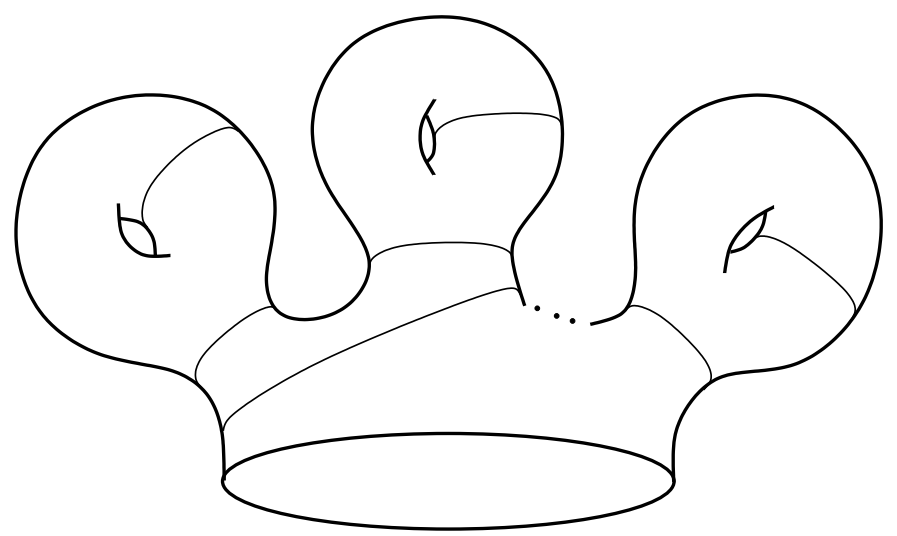

Figure 3: A stabilization glued into $\Sigma$

the resulting Heegaard splitting. Note that exactly $(h-g)$ of the new loops are nonseparating and the rest of the loops bound disks in both $H_{1}^{\prime}$ and $H_{2}^{\prime}$. The vertex $v_{1}^{\prime} \in C^{*}\left(\Sigma^{\prime}\right)$ given by the loops $k_{1}^{1}, \ldots, k_{1}^{m^{\prime}}$ defines the compression body $H_{1}^{\prime}$.

We will create a sequence of vertices $v_{1}^{\prime}, \ldots, v_{n^{\prime \prime}}^{\prime}$ in $C^{*}\left(\Sigma^{\prime}\right)$ such that $n^{\prime \prime} \leq 2 n+(h-g)$ and $v_{n^{\prime \prime}}^{\prime}$ defines $H_{2}^{\prime}$. If $l_{1}^{1}=l_{2}^{1}$ then for $j \leq m$, let $k_{2}^{j}$ be the loop in $\Sigma^{\prime}$ defined by $l_{2}^{j}$ in $\Sigma$ and for $j>m$, let $k_{2}^{j}=k_{1}^{j}$. One can check that the vertex $v_{2}^{\prime} \in C^{*}\left(\Sigma^{\prime}\right)$ is connected to $v_{1}^{\prime}$ by an edge.

If $l_{1}^{1} \neq l_{2}^{1}$, then this construction does not work because $l_{2}^{1}$ will intersect $k_{1}^{m+1}$. We need to get $k_{1}^{m+1}$ out of the way first. Let $k_{2}^{j}=k_{1}^{j}$ for each $j \neq m+1$. The loop $k_{1}^{m+1}$ sits in a four-punctured sphere. Two of the punctures come from $l_{1}^{1}$ and $k_{2}^{m+2}$. Let $l_{1}^{j}$ be the loop defining one of the two remaining punctures. Let $k_{2}^{m+1}$ be a loop parallel to $l_{1}^{j}$ so that $k_{2}^{m+2}$ is in the annulus defined by $k_{2}^{m+1}$ and $l_{1}^{j}$. (See Figure 2.) The vertex $v_{2}^{\prime}$ defined by these loops is an edge away from $v_{1}^{\prime}$.

Now we can define $k_{3}^{j}$ almost as we defined $k_{2}^{j}$ in the original case. Let $k_{3}^{j}=l_{2}^{j}$ for $1<j \leq m$ and $k_{3}^{j}=k_{2}^{j}$ for $j>m$. We cannot necessarily choose $k_{3}^{1}$ to be equal to $l_{2}^{1}$ because $l_{2}^{1}$ may intersect $k_{2}^{m+1}$. However, the image in $\Sigma$ of $k_{2}^{m+1}$ is parallel to a loop which is disjoint from $l_{2}^{1}$. We can let $l_{3}^{1}$ be a loop in $\Sigma^{\prime}$ which is disjoint from $k_{2}^{m+1}$ and whose image in $\Sigma$ is isotopic to $l_{2}^{1}$. The vertex $v_{3}^{\prime}$ defined by these loops is an edge away from $v_{2}^{\prime}$.

The vertex $v_{2}^{\prime}$ or $v_{3}^{\prime}$ defined in this way has the property that if we surger $\Sigma^{\prime}$ along $k_{2}^{m+2}$, the loops in the component isotopic to $\Sigma$ define the vertex $v_{2}$. Thus we can 
repeat the construction for $v_{3}$ through $v_{n}$. At each stage, if the extra loop is parallel to a loop that needs to be moved, it takes one extra move to push it out of the way. The resulting sequence $v_{1}^{\prime}, \ldots, v_{n^{\prime}}^{\prime}$ will be at most twice as long as the original.

There are only $h-g$ moves left before $v_{i}^{\prime}$ defines the handlebody $H_{2}^{\prime}$. Exactly $h-g$ of the loops $k_{n^{\prime}}^{m+3}, \ldots, k_{n^{\prime}}^{m^{\prime}}$ do not bound disks in $H_{2}$. However, each can be replaced by a new loop, disjoint from the rest, which does bound a disk in $H_{2}$. Thus in $h-g$ moves, we can complete the sequence $v_{1}^{\prime}, \ldots v_{n^{\prime \prime}}^{\prime}$ so that each loop $k_{n^{\prime \prime}}^{m+3}, \ldots, k_{n^{\prime \prime}}^{m+3}$ bounds a disk in $H_{2}$. By construction, the loop $k_{n^{\prime \prime}}^{m+2}=k_{1}^{m+2}$ bounds a disk in $H_{2}$ and the loop $k_{n^{\prime \prime}}^{m+1}$ is parallel to some $l_{n}^{j}$ in $\Sigma$ so it bounds a disk in $H_{2}$. Thus $v_{n^{\prime}}^{\prime}$ defines $H_{2}^{\prime}$.

By carefully choosing the sequence of loops $l_{j}^{i}$ to which $k_{m+1}^{i^{\prime}}$ is parallel, one could improve the bound, but for our purposes, the existence of a bound is all that is necessary. The bound is true for every $h$, but with $g$ fixed, so the sequence $A_{h}(\Sigma)$ is bounded above and below as $h \rightarrow \infty$. We will show that the sequence actually converges.

Lemma 16 For sufficiently large $g, A_{h}(\Sigma) \leq A_{g}(\Sigma)$ and $A_{h}^{P}(\Sigma) \leq A_{g}^{P}(\Sigma)$ whenever $h \geq g$.

Proof Let $g^{\prime}$ be the genus of $\Sigma$. By Lemma $15, A_{g}(\Sigma)$ is bounded by $2 A_{g^{\prime}}(\Sigma)+$ $\left(g^{\prime}-b\right)$. Choose $g$ so that $4 g-3>2 A_{g^{\prime}}(\Sigma)+\left(g^{\prime}-b\right)$. Then $A_{g}(\Sigma)<4 g-3$ so $D\left(\Sigma_{g}\right)<3 g-3$. This implies that for the minimal path from $H_{1}$ to $H_{2}$ in $C^{*}\left(\Sigma_{g}\right)$, there is some loop that is not moved.

Consider the proof of Lemma 15. If we had chosen $l_{1}$ to be a loop that is never moved in the sequence $v_{1}, \ldots, v_{n}$, then for the path $v_{1}^{\prime}, \ldots, v_{n^{\prime}}^{\prime}$, we would have $n^{\prime}=n+(h-g)$. For sufficiently large g, we can find such a loop, so we have $D\left(\Sigma_{h}\right) \leq D\left(\Sigma_{g}\right)+(h-g)$. This proves the lemma.

Main Theorem 17 The limits $\lim _{g \rightarrow \infty} A_{g}(\Sigma)$ and $\lim _{g \rightarrow \infty} A_{g}^{P}(\Sigma)$ exist and depend only on $M$, not on the choice of $\Sigma$.

Proof The sequence $A_{g}(\Sigma)$ is bounded below by Lemma 8 and non-increasing for sufficiently large $g$ by Lemma 16, so the limit exists.

Given two Heegaard surfaces $\Sigma$ and $\Sigma^{\prime}$ of $M$, there is a common stabilization. In other words, there is a genus $g$ and a Heegaard surface $\Sigma^{\prime \prime}$ such that $\Sigma^{\prime \prime}$ is isotopic to $\Sigma_{g}$ and $\Sigma_{g}^{\prime}$. Then $A_{h}(\Sigma)=A_{h}\left(\Sigma^{\prime \prime}\right)=A_{h}\left(\Sigma^{\prime}\right)$ for $h \geq g$ and the limits are the same for $A_{h}(\Sigma)$ and $A_{h}\left(\Sigma^{\prime}\right)$ 
Definition 18 The Heegaard complexity of $M$ is $A(M)=\lim _{h \rightarrow \infty} A_{h}(\Sigma)$ where $\Sigma$ is any Heegaard splitting of $M$. The pants complexity of $M$ is $A^{P}(M)=$ $\lim _{h \rightarrow \infty} A_{h}^{P}(\Sigma)$.

The sequence $A_{h}(\Sigma)$ consists entirely of integers so for any $\Sigma, A_{h}(\Sigma)=A(M)$ for some $h$. Thus if $A(M)=-n$ then $M=S^{3}$ or $M$ is a connect sum of compression bodies, lens spaces and copies of $S^{1} \times S^{3}$.

\section{Properties}

Lemma 19 Let $M$ and $M^{\prime}$ be compact manifolds. Then $A\left(M \# M^{\prime}\right) \leq A(M)+$ $A\left(M^{\prime}\right)$ and $A^{P}\left(M \# M^{\prime}\right) \leq A^{P}(M)+A^{P}\left(M^{\prime}\right)$

(Here, $M \# M^{\prime}$ is the connect sum of $M$ and $M^{\prime}$.)

Proof Let $\Sigma$ be a Heegaard splitting of $M$. Let $\Sigma_{g}$ be a stabilization such that $D\left(\Sigma_{g}\right)+b-g=A(M)$ and for a minimal path from $H_{1}$ to $H_{2}$ in $C^{*}\left(\Sigma_{g}\right)$, there is a loop $l_{1}$ which is a vertex of each $\sigma_{v_{i}}$. Let $\Sigma_{g^{\prime}}^{\prime}$ be a similar Heegaard splitting for $M^{\prime}$ and let $l_{1}^{\prime}$ be the loop that is fixed.

We will construct a Heegaard splitting for $M \# M^{\prime}$ as follows: Let $l_{2}$ be a loop in $\Sigma_{g}$ parallel to $l_{1}$, let $B$ be a ball in $M$ such that $M \cap \Sigma_{g}$ is a disk in the annulus defined by $l_{1}$ and $l_{2}$ and define $l_{3}=\partial\left(B \cap \Sigma_{g}\right)$. Define $l_{2}^{\prime}, l_{3}^{\prime}$ and $B^{\prime}$ similarly in $M^{\prime}$.

Take the connect sum of $M$ and $M^{\prime}$ by removing $B$ and $B^{\prime}$ from $M$ and $M^{\prime}$ respectively, then gluing together the resulting boundaries. Choose a gluing map that sends $l_{3}$ to $l_{3}^{\prime}$. Let $\Sigma^{\prime \prime}$ be the resulting Heegaard splitting. The paths in $C^{*}\left(\Sigma_{g}\right)$ and $C^{*}\left(\Sigma_{g}^{\prime}\right)$ define a path in $C^{*}\left(\Sigma^{\prime \prime}\right)$, implying that. $D\left(\Sigma^{\prime \prime}\right) \leq D\left(\Sigma_{g}\right)+D\left(\Sigma_{g^{\prime}}\right)$ and the genus of $\Sigma^{\prime \prime}$ is $g+g^{\prime}$.

The sequence of stabilizations $\Sigma_{h}^{\prime \prime}$ can be constructed by the above gluing operation, by taking stabilizations of $\Sigma_{g}$. Thus $D\left(\Sigma_{g+g^{\prime}+i}^{\prime \prime}\right) \leq D\left(\Sigma_{g+i}\right)+D\left(\Sigma_{g^{\prime}}\right)$. Because $D\left(\Sigma_{g+i}\right)=D\left(\Sigma_{g}\right)$ for all $i$, we have $A\left(M \# M^{\prime}\right) \leq A(M)+A\left(M^{\prime}\right)$.

The converse statement is an open question: Is it necessarily true that $A\left(M \# M^{\prime}\right) \geq$ $A(M)+A\left(M^{\prime}\right)$ ?

Lemma 20 Let $M$ be a manifold with boundary and let $M^{\prime}$ be the result of filling one or more torus boundary components with solid tori. Then $A\left(M^{\prime}\right) \leq A(M)$. 
Proof Let $\Sigma$ be a Heegaard surface for $M$ and let $\Sigma^{\prime}$ be the image of $\Sigma$ in the induced map $M \rightarrow M^{\prime}$. This $\Sigma^{\prime}$ is a Heegaard surface for $M^{\prime}$. Let $v_{1}, \ldots, v_{n}$ be a path in $C^{*}(\Sigma)$ from $H_{1}$ to $H_{2}$. The map $\Sigma \rightarrow \Sigma^{\prime}$ suggests an isomorphism $C^{*}(\Sigma) \rightarrow C^{*}\left(\Sigma^{\prime}\right)$. Let $v_{1}^{\prime}, \ldots, v_{n}^{\prime}$ be the images of $v_{1}, \ldots, v_{n}$.

The vertex $v_{n}$ defines $H_{2}^{\prime}$ because $H_{2}$ is a handlebody so $H_{2}^{\prime}$ is the image of $H_{2}$ in the induced map. However, $v_{1}$ may not define $H_{1}$ because for each torus boundary component that is filled, there is a loop in $\Sigma$ parallel to $\partial_{-} H_{1}$ which may not bound a disk in $H_{1}^{\prime}$.

Let $T$ be a torus boundary component which is filled so that the boundary of a meridian disk maps to a loop $\alpha \subset T$. If we cut $H_{1}$ along the maximal collection disks defined by the pants decomposition, there will be a loop $l_{1}$ on the component containing $T$ and a scar bound by a loop $l_{2}$. The loop $\alpha$ (which sits in $\partial_{-} H_{1}$ ) can be projected into $\partial_{+} H_{1}$ so that its image sits in the torus with $l_{1}$ and is disjoint from $l_{2}$. Thus it takes one move to replace $l_{1}$ with the image of $\alpha$.

The image of $\alpha$ bounds a disk in $H_{1}^{\prime}$. For each torus component which is filled, it takes at most one move to replace a loop in the pants decomposition for $H_{1}$ with a loop bounding a disk in $H_{1}^{\prime}$. The final product is a pants decomposition containing a maximal collection of disks for $H_{1}^{\prime}$. The sum $b^{\prime}$ of the genera of the boundary components goes down by one for every Dehn filling.

Since we have $A_{g}\left(\Sigma^{\prime}\right)=D\left(\Sigma_{g}^{\prime}\right)-g+b^{\prime}$, we know that $A_{g}\left(\Sigma^{\prime}\right) \leq A_{g}(\Sigma)$ when $g$ is the genus of $\Sigma$. The same proof works for every stabilization of $\Sigma$ so in the limit we have $A\left(M^{\prime}\right) \leq A(M)$.

The equivalent statement is not true for $A^{P}(M)$ because there is no control over the number of times the image of the loop $\alpha$ intersects the loop $l_{1}$.

Although the Heegaard complexity cannot increase under Dehn filling, it can drop by an arbitrary amount. In particular, if $M$ is the complement of a knot in $S^{3}$, then there is a Dehn filling which produces $M^{\prime}=S^{3}$, so $A\left(M^{\prime}\right)=0$, regardless of the Heegaard complexity of $M$.

Lemma 21 Let $\left(\Sigma, H_{1}, H_{2}\right)$ be an irreducible Heegaard splitting of a closed manifold $M$ and let $g$ be the genus of $\Sigma$. Then $D(\Sigma) \geq 3 g-3$.

Proof Let $v_{0}, \ldots, v_{n}$ be a path in $C^{*}(\Sigma)$ such that $v_{1}$ defines $H_{1}, v_{n}$ defines $H_{2}$ and $n=D(\Sigma)$. Both $H_{1}$ and $H_{2}$ are handlebodies so if $\sigma_{v_{1}}$ and $\sigma_{v_{n}}$ share a vertex $u \in C(\Sigma)$ then $u$ corresponds to a loop in $\Sigma$ that bounds a disk in $H_{1}$ and a disk in $\mathrm{H}_{2}$.

Algebraic 83 Geometric Topology, Volume 6 (2006) 
Since $\Sigma$ is irreducible, there is no such loop in $\Sigma$ so $\sigma_{v_{0}}$ and $\sigma_{v_{n}}$ cannot share a vertex. The simplex $\sigma_{v_{0}}$ has $3 g-3$ vertices and consecutive simplices $\sigma_{v_{i}}, \sigma_{v_{i+1}}$ share all but one vertex so there must be at least $3 g-2$ in the sequence. Thus it must be that $n \geq 3 g-2$ and $D(\Sigma) \geq 3 g-3$.

Lemma 22 Let $\left(\Sigma, H_{1}, H_{2}\right)$ be a strongly irreducible Heegaard splitting of a closed manifold $M$ and let $g$ be the genus of $\Sigma$. Then $D(\Sigma) \geq 6 g-7$.

We will sketch the proof, since the result is not vital to the rest of the paper. Because $\Sigma$ is irreducible, each of the loops must be moved at least once. Let $l$ be the last loop that's moved. A loop $l^{\prime}$ which is moved before $l$ is replaced by a loop $l^{\prime \prime}$ disjoint from $l$. Since $l$ bounds a disk in $H_{1}, l^{\prime \prime}$ cannot bound a disk in $H_{2}$ (since $\Sigma$ is strongly irreducible) so $l^{\prime \prime}$ must be moved later on. Every loop other than $l$ must therefore be moved at least twice (once before $l$ and once after) so $D(\Sigma) \geq 6 g-7$.

Unfortunately, once the Heegaard surface $\Sigma$ is replaced with a stabilization $\Sigma^{\prime}$, the distance may drop by an arbitrary amount. For example, Kobayashi [5] has constructed a manifold, based on work by Casson and Gordon, with a sequence of strongly irreducible Heegaard splittings of arbitrarily high genus. Sedgwick [8] later showed that the result of stabilizing any of these once is also a stabilization of all the lower genus splittings.

By Lemma 22, the unstabilized Heegaard splittings have arbitrarily high distance, but by Lemma 15, the distances of the stabilizations are bounded, so the distance must fall by an arbitrarily large amount after stabilization.

There is still a relationship between the Heegaard genus of the manifold and the Heegaard complexity, but it is not as strong.

Lemma 23 If $M$ is irreducible and $\partial M=\varnothing$ then the Heegaard genus of $M$ is less than or equal to $\frac{A(M)+2}{2}$.

Proof Let $\Sigma$ be a Heegaard splitting of $M$ such that $A_{g}(\Sigma)=A(M)$, where $g$ is the genus of $\Sigma$. Let $v_{1}, \ldots, v_{n}$ be a path in $C^{*}(\Sigma)$ of length $D(\Sigma)$. Let $\mathbf{L}$ be the loops in $\Sigma$ defined by the vertices of $\sigma_{v_{1}}$. By choosing $g$ large enough, we can assume there is at least one loop $l_{i}$ which also bounds a disk in $\mathrm{H}_{2}$.

Let $\mathbf{L}^{\prime}$ be the collection of loops which are never moved. In other words if $l_{j} \in \mathbf{L}^{\prime}$ then for every vertex $v_{i}$ in the sequence, $l_{j}$ corresponds to a vertex of $\sigma_{v_{i}}$. In particular, $l_{j}$ is a vertex of $\sigma_{v_{1}}$ and of $\sigma_{v_{n}}$. Thus $l_{j}$ bounds a disk $D_{j}^{1} \subset H_{1}$ and a disk $D_{j}^{2} \subset H_{2}$ (since $H_{1}$ and $H_{2}$ are handlebodies). We can choose the disks corresponding to the loops in $\mathbf{L}$ so that the collection is pairwise disjoint. Thus each $l_{j}$ suggests a disjoint, embedded 2-sphere $S_{j}$.

Algebraic $8 \mathcal{G}$ Geometric Topology, Volume 6 (2006) 
Because $M$ is irreducible, each sphere $S_{j}$ bounds a ball $B_{j} \subset M$. Let $\Sigma^{\prime}=\Sigma \backslash \bigcup B_{j}$. This is a punctured surface. Disks can be glued into the punctures to make $\Sigma^{\prime}$ a Heegaard surface for $M$, so the genus of $\Sigma^{\prime}$ is at least the Heegaard genus of $M$. Let $h$ be the genus of $\Sigma^{\prime}$. Some subset $\mathbf{L}^{\prime \prime}$ of the loops $\mathbf{L}$ form a pair-of-pants decomposition for $\Sigma^{\prime}$. This is a punctured surface of genus $h$ (there may be more than one puncture) so there are at least $3 h-2$ loops in $\mathbf{L}^{\prime \prime}$ and none of these loops are in $\mathbf{L}^{\prime}$.

In the surface $\Sigma \backslash \Sigma^{\prime}$, there are at least $g-h$ non-separating loops. Since $M$ is irreducible, none of these loops can be in $\mathbf{L}^{\prime}$ so there are at least $g-h$ more loops that are moved. There are at least $2 h-2+g$ loops in $\mathbf{L} \backslash \mathbf{L}^{\prime}$ so $D(\Sigma) \geq 2 h-2+g$ and $A_{g}(\Sigma) \geq 2 h-2$. This is true of every stabilization of $\Sigma$ so $h \leq \frac{A(M)+2}{2}$ and the Heegaard genus of $M$ is at most $h$.

Corollary 24 For every positive integer $N$, there is a manifold $M$ with $A(M)>N$.

\section{Comparing manifolds}

Let $M$ and $M^{\prime}$ be compact, connected, orientable 3-manifolds such that $\partial M=\partial M^{\prime}$. (Both boundaries may be empty.) Let $\left(\Sigma, H_{1}, H_{2}\right)$ and $\left(\Sigma^{\prime}, H_{1}^{\prime}, H_{2}^{\prime}\right)$ be Heegaard splittings of $M, M^{\prime}$ respectively such that $\mathrm{H}_{2}$ and $\mathrm{H}_{2}^{\prime}$ are handlebodies of the same genus.

Let $\phi: H_{1} \rightarrow H_{1}^{\prime}$ be any homeomorphism. Such a map exists because $\partial_{-} H_{1}=\partial M=$ $\partial M^{\prime}=\partial_{-} H_{1}^{\prime}$. This induces a homeomorphism from $\Sigma=\partial_{+} H_{1}$ to $\Sigma^{\prime}=\partial_{+} H_{1}^{\prime}$ and this homeomorphism suggests an isomorphism $\widehat{\phi}: C^{*}(\Sigma) \rightarrow C^{*}\left(\Sigma^{\prime}\right)$ or $\widehat{\phi}: C^{P}(\Sigma) \rightarrow$ $C^{P}\left(\Sigma^{\prime}\right)$.

Definition 25 The dual distance between the two Heegaard splittings is $D\left(\Sigma, \Sigma^{\prime}\right)=$ $\left\{D\left(v, v^{\prime}\right): v\right.$ defines $H_{2}, \widehat{\phi}\left(v^{\prime}\right)$ defines $\left.H_{2}^{\prime}\right\}$. The pants distance is $D^{P}\left(\Sigma, \Sigma^{\prime}\right)=$ $\min \left\{D^{P}\left(v, v^{\prime}\right): v\right.$ defines $H_{2}, \widehat{\phi}\left(v^{\prime}\right)$ defines $\left.H_{2}^{\prime}\right\}$. Both minima are taken over all homeomorphisms $\phi: H_{1} \rightarrow H_{1}^{\prime}$.

In other words, identify $H_{1}$ and $H_{1}^{\prime}$ and consider two pants decompositions of $\partial_{+} H_{1}$ such that one is a Heegaard diagram for $M$ and the other is a Heegaard diagram for $M^{\prime}$. The value of $D\left(\Sigma, \Sigma^{\prime}\right)$ is the smallest possible dual distance between any two such pants decompositions.

As in Section 5, consider a sequence $\Sigma_{g}$ of genus- $g$ stabilizations of $\Sigma$ and a sequence $\Sigma_{g}^{\prime}$ of stabilizations of $\Sigma^{\prime}$.

Algebraic $8 \mathcal{G}$ Geometric Topology, Volume 6 (2006) 
Lemma $26 D\left(\Sigma_{g}, \Sigma_{g}^{\prime}\right) \leq 2 D\left(\Sigma, \Sigma^{\prime}\right)$.

The proof is almost identical to the proof of Lemma 15, with the exception that there are not $(h-g)$ loops left at the end which need to be moved. The proof is left to the reader, as is the proof of the following lemma.

Lemma 27 For sufficiently large $g, D\left(\Sigma_{h}, \Sigma_{h}^{\prime}\right) \leq D\left(\Sigma_{g}, \Sigma_{g}^{\prime}\right)$ whenever $h \geq g$.

Theorem 28 The sequence $D\left(\Sigma_{g}, \Sigma_{g}^{\prime}\right)$ converges and is independent of the choices of $\Sigma$ and $\Sigma^{\prime}$.

Proof The distance $D\left(\Sigma_{g}, \Sigma_{g}^{\prime}\right)$ is non-negative and the sequence is non-increasing for sufficiently large $g$, so it converges. Showing that the limit is unique is slightly more tricky.

Let $\left(\Sigma^{1}, H_{1}^{1}, H_{2}^{1}\right)$ and $\left(\Sigma^{2}, H_{1}^{2}, H_{2}^{2}\right)$ be Heegaard splittings of $M$. We know that there is a stabilization $\left(\Sigma^{3}, H_{1}^{3}, H_{2}^{3}\right)$ of $\Sigma^{1}$ and a stabilization $\left(\Sigma^{4}, H_{1}^{4}, H_{2}^{4}\right)$ of $\Sigma^{2}$ such that $\Sigma^{3}$ is isotopic to $\Sigma^{4}$. If $M$ has non-empty boundary then this isotopy must send $H_{1}^{3}$ to $H_{1}^{4}$ since $H_{2}^{4}$ is a handlebody but $H_{1}^{3}$ is not.

However, if $M$ is closed, the isotopy could send $H_{1}^{3}$ to $H_{2}^{4}$. Since the definition of $D\left(\Sigma, \Sigma^{\prime}\right)$ distinguishes between $H_{1}$ and $H_{2}$, the distances $D\left(\Sigma^{3}, \Sigma^{\prime}\right)$ and $D\left(\Sigma^{4}, \Sigma^{\prime}\right)$ may not be equal.

Implicit in most proofs of the stabilization theorem it is also proven that the stabilizations can be chosen so that an isotopy sends $\Sigma^{3}$ to $\Sigma^{4}$ and sends $H_{1}^{3}$ to $H_{1}^{4}$. (See, for example, Rubinstein and Scharlemann's proof [7].) In this case, it must be the case that $D\left(\Sigma^{3}, \Sigma^{\prime}\right)=D\left(\Sigma^{4}, \Sigma^{\prime}\right)$. So the limit of the distances is independent of the choice of $\Sigma$. A similar consideration for $M^{\prime}$ shows that the distance is independent of the choice of $\Sigma^{\prime}$, and therefore the limit depends only on $M$ and $M^{\prime}$.

Definition 29 We define the Heegaard distance $D\left(M, M^{\prime}\right)$ to be the limit of the sequence $D\left(\Sigma_{g}, \Sigma_{g}^{\prime}\right)$ for any Heegaard surfaces $\Sigma$ and $\Sigma^{\prime}$ of $M$ and $M^{\prime}$.

It is immediate that $D\left(M, M^{\prime}\right)=0$ if and only if $M=M^{\prime}$. When $M \neq M^{\prime}$, there is a very simple description of $D\left(M, M^{\prime}\right)$ as follows: Let $K \subset M$ be a link. We will say that $M$ and $M^{\prime}$ are connected by $K$ if $M^{\prime}$ is the result of some Dehn surgery on $K \subset M$. Let $c\left(M, M^{\prime}\right)$ be the smallest integer $c$ such that $M$ and $M^{\prime}$ are connected by an $c$-component link.

Theorem $30 \quad D\left(M, M^{\prime}\right)=c\left(M, M^{\prime}\right)$. 
Proof We will first show that $D\left(M, M^{\prime}\right) \leq c\left(M, M^{\prime}\right)$. Let $K \subset M$ be a $c$-component link in $M$ such that some Dehn surgery on $K$ yields $M^{\prime}$. Let $\left(\Sigma, H_{1}, H_{2}\right)$ be a Heegaard splitting of $M$ such that there is a trivalent spine $G$ of $H_{2}$ in which each component of $K$ appears as an edge of $G$ with both ends on the same vertex.

Let $l_{1}, \ldots, l_{m}$ be loops on $\Sigma$ defined by the meridian disks dual to the edges of $G$ and assume $l_{1}, \ldots, l_{n}$ correspond to the edges of $G$ defined by the components of $K$. Since $G$ is trivalent, the loops suggest a pants decomposition of $\Sigma$ and the corresponding vertex $v_{0}$ of $C^{*}(\Sigma)$ defines $H_{2}$.

The loop $l_{1}$ defines a meridian curve on a component of $K$. Let $l_{1}^{\prime}$ be the meridian defined by the Dehn surgery on $K$. This loop is disjoint from the loops $l_{2}, \ldots, l_{m}$ because of the way we chose $G$. Thus the vertex $v_{1}$ defined by $l_{1}^{\prime}, l_{2}, \ldots, l_{m}$ is connected to $v_{0}$ by an edge in $C^{*}(\Sigma)$.

Continuing in this way for the loops $l_{2}, \ldots, l_{n}$, we can construct a path $v_{0}, \ldots, v_{n}$ where $v_{n}$ is defined by the loops $l_{1}^{\prime}, \ldots, l_{n}^{\prime}, l_{n+1}, \ldots, l_{m}$. By the construction, this vertex will define a handlebody in a Heegaard splitting $\left(\Sigma^{\prime}, H_{1}^{\prime}, H_{2}^{\prime}\right)$ of $M^{\prime}$. For any stabilization of $\left(\Sigma, H_{1}, H_{2}\right)$, we can construct a graph with the same properties as $G$. Thus $D\left(\Sigma_{g}, \Sigma_{g}^{\prime}\right) \leq c$ for every $g$ and $D\left(M, M^{\prime}\right) \leq c$.

We now see a connection between Heegaard distance and Dehn surgery. To prove that $D\left(M, M^{\prime}\right) \geq c$, we need the following Lemma:

Lemma 31 If $D\left(M, M^{\prime}\right)=1$ then $M$ and $M^{\prime}$ are connected by a knot (a onecomponent link).

Proof Let $\left(\Sigma, H_{1}, H_{2}\right)$ and $\left(\Sigma^{\prime}, H_{1}^{\prime}, H_{2}^{\prime}\right)$ be Heegaard splittings for $M, M^{\prime}$ respectively such that $D\left(\Sigma, \Sigma^{\prime}\right)=1$. This means there is a pants decomposition $l_{1}, l_{2}, \ldots, l_{m}$ for $\partial H_{1}$ giving a Heegaard diagram for $M$ such that replacing $l_{1}$ with $l_{1}^{\prime}$ creates a pants decomposition $l_{1}^{\prime}, l_{2}, \ldots, l_{m}$ giving a Heegaard diagram for $M^{\prime}$.

The loop $l_{1}$ bounds a disk in $H_{2}$ and the loop $l_{1}^{\prime}$ bounds a disk in $H_{2}^{\prime}$. As in Section $2, l_{1}$ may sit in a four-punctured sphere or a once-punctured torus, and $l_{1}^{\prime}$ will lie in the same type of component. If $l_{1}$ sits in a four-punctured sphere, then the punctured sphere and the disks defined by the punctures bound a ball in $H_{2}$. Since $l_{1}^{\prime}$ is on the boundary of this ball, it bounds a disk in $H_{2}$. Thus $l_{1}^{\prime}, l_{2}, \ldots, l_{m}$ is a Heegaard diagram for $M$ as well as $M^{\prime}$ so the two manifolds are homeomorphic. Since $D\left(M, M^{\prime}\right) \neq 0$, the manifolds are distinct, and $l_{1}$ must sit in a punctured torus.

The punctured torus and the disk defined by the puncture bound a solid torus in $M$ and a solid torus in $M^{\prime}$. The remainder of the Heegaard diagrams are identical so the 
complements of the solid tori in $M$ and $M^{\prime}$ are homeomorphic. We get $M$ and $M^{\prime}$ by gluing solid tori to the boundaries of the complement, so Dehn surgery on the solid torus in $M$ will yield $M^{\prime}$.

We can now finish the proof of Theorem 30

Let $v_{1}, \ldots, v_{n}$ be a path in $C^{*}(\Sigma)$ from $H_{2}$ to $H_{2}^{\prime}$. Each vertex $v_{i}$ defines a Heegaard splitting of a manifold $M_{i}$. Because the Heegaard distance between consecutive manifolds is at most 1, there is a sequence of knots such that Dehn surgery on each knot yields the next manifold. By keeping track of the images of these knots in $M$, we find a link with at most as many components as the distance of the path. Thus $c\left(M, M^{\prime}\right) \leq D\left(M, M^{\prime}\right)$ and the proof is complete.

As one might expect, there is an analogous Theorem for $D^{P}\left(M, M^{\prime}\right)$. Let $c^{1}\left(M, M^{\prime}\right)$ be the minimum number of components of a link $K \subset M$ such that $M^{\prime}$ is the result of a surgery in which a meridian of each component is replaced by a loop which intersects the meridian once.

Theorem $32 D^{P}\left(M, M^{\prime}\right)=c^{1}\left(M, M^{\prime}\right)$.

The proof is almost identical to the proof of Theorem 30 and will be left to the reader.

\section{Questions and speculations}

Question 1 Is $A(M)$ or $A^{P}(M)$ related to (quasi-equal to?) a manifold invariant which is already known?

The most tempting possibility is that for hyperbolic manifolds, the complexity could be related to volume. Juan Souto [9] has announced joint work with Jeff Brock showing that in certain cases, the pants distance of a specific Heegaard splitting is quasi-isometric to its hyperbolic volume. Brock [1] has previously proven results relating distance in the pants complex to volumes of convex cores.

Another possibility is that the pants complexity could be related to the number of tetrahedra needed for a triangulation of $M$, or to triangulate a 4-manifold bounded by $M$. By Hatcher and Thurston, an edge path in the pants complex suggests a smooth path in $C^{\infty}(\Sigma)$. Applying these functions to the level surfaces of a sweep-out of the Heegaard splitting suggests a stable function from the manifold to $\mathbb{R}^{2}$. Costantino and Thurston [2] have used 2-dimensional stable functions to relate the number of tetrahedra in a triangulation of $M$ to the number of tetrahedra in a 4-manifold which it bounds. The link between the complexity of the stable function and triangulations may be applicable to the stable function induced by a Heegaard splitting. 
Question 2 Is there any way to calculate the complexity?

This is already known to be a very difficult problem. There is currently a lot of work being done to calculate the distance between two points in the pants complex or the Hempel distance of a Heegaard splitting. Calculating the Heegaard complexity should be even more difficult because it requires calculating the distance for an infinite number of splittings.

Question 3 Is either complexity additive under connect summing?

This is a deceptively simple-sounding problem. We have seen that both complexities are sub-additive. Unlike the Hempel distance, which is zero when $M$ is reducible, the dual distance or pants distance may ignore the reducing disks when finding a shortest path.

Question 4 What conditions will guarantee that $A^{P}(M)$ will not increase after Dehn filling a torus boundary component? What conditions will guarantee that $A(M)$ will not decrease?

It was pointed out earlier that there is no analogy to Lemma 20 for $A^{P}(M)$. Given a manifold with boundary, a Heegaard splitting and a minimal distance path in $C^{P}(\Sigma)$, the initial pants decomposition of $\Sigma$ will define loops in $\partial M$. Dehn fillings which take into account these loops will guarantee that $D^{P}(\Sigma)$ does not increase. The question is whether or not there is a way to predict these loops from the topology of the manifold, ie without calculating geodesic paths for an infinite sequence of Heegaard splittings.

Question 5 How does the complexity behave under finite covers?

Lackenby [6] has shown that the asymptotic behavior of the Heegaard genus of finite covers is related to Thurston's virtually Haken conjecture and virtually fibered conjecture. Essentially, if the Heegaard genera of finite covers of a given manifold are bounded by a nice enough function of the degree of the covers then one of the covers must be Haken or fibered.

Given a finite cover $M^{\prime}$ of $M$ and a Heegaard splitting, there is an induced Heegaard splitting of $M^{\prime}$. By Lemma 23, rather than having to find an alternative splitting of $M$ with lower genus, it would only be necessary to show that the pants distance is bounded by a nice enough function of the degree of the cover.

Question 6 If $M$ and $N$ are irreducible 3-manifolds and there is a degree-one map from $M$ to $N$, does this imply that $A(N) \leq A(M)$ ? 
Waldhausen [10] showed that given a degree-one map $f: M \rightarrow N$ and a Heegaard splitting $\left(\Sigma, H_{1}, H_{2}\right)$ of $N$, one can construct a Heegaard splitting $\left(\Sigma^{\prime}, H_{1}^{\prime}, H_{2}^{\prime}\right)$ of $M$ such that $f$ maps $H_{1}^{\prime}$ and $H_{2}^{\prime}$ onto $H_{1}$ and $H_{2}$, respectively, by a simple type of degree-one map. Given a path in $C^{*}(\Sigma)$, it may be possible to construct a path in $C^{*}\left(\Sigma^{\prime}\right)$ which is, in some sense, induced by the map $f$.

A positive answer to this question would imply the Poincare conjecture because a homotopy equivalence is a degree-one map. So, if $M$ is $S^{3}$ then $A(M)=0$ so $A(N)=0$ and $N$ is $S^{3}$. If we remove the assumption that $M$ is irreducible then the answer is no, since there is a degree-one map from $S^{1} \times S^{2}$ to $S^{3}$.

\section{References}

[1] J F Brock, The Weil-Petersson metric and volumes of 3-dimensional hyperbolic convex cores, J. Amer. Math. Soc. 16 (2003) 495-535 MR1969203

[2] F Costantino, D Thurston, 3-manifolds efficiently bound 4-manifolds, preprint (2005)

[3] A Hatcher, W Thurston, A presentation for the mapping class group of a closed orientable surface, Topology 19 (1980) 221-237 MR579573

[4] J Hempel, 3-manifolds as viewed from the curve complex, Topology 40 (2001) 631-657 MR1838999

[5] T Kobayashi, A construction of 3-manifolds whose homeomorphism classes of Heegaard splittings have polynomial growth, Osaka J. Math. 29 (1992) 653-674 MR1192734

[6] M Lackenby, The asymptotic behaviour of Heegaard genus, Math. Res. Lett. 11 (2004) 139-149 MR2067463

[7] H Rubinstein, M Scharlemann, Comparing Heegaard splittings of non-Haken 3manifolds, Topology 35 (1996) 1005-1026 MR1404921

[8] E Sedgwick, An infinite collection of Heegaard splittings that are equivalent after one stabilization, Math. Ann. 308 (1997) 65-72 MR1446199

[9] J Souto, personal correspondence (2005)

[10] F Waldhausen, On mappings of handlebodies and of Heegaard splittings, from: “Topology of Manifolds (Georgia, 1969)”, Markham, Chicago, Ill. (1970) 205-211 MR0271941

Mathematics Department, University of California

Davis, CA 95616, USA

jjohnson@math.ucdavis.edu

Received: 5 May 2006

Algebraic $6 \mathcal{G}$ Geometric $\mathcal{T}$ opology, Volume 6 (2006) 